Abhandlung

Prof. Dr. Martin Heger* und Wiss. Mitarbeiter Michael Jahn

\title{
Den Helfern zu Hilfe: Verbesserter Schutz für professionelle zivile Helfer durch $\S 305$ a und $\S 114$ Abs. 3 StGB?
}

DOI 10.1515/juru-2015-0065

Fachverbände von Feuerwehr und Rettungsdiensten haben seit langem gefordert, dass ihre Einsatzkräfte in Notfallsituationen unter besonderen Schutz gestellt werden. Mit dem 44. StrÄndG hat sich der Gesetzgeber dieser Forderung schließlich angenommen und das Strafgesetzbuch um entsprechende Regelungen ergänzt. Diese Regelungen verbessern die Situation ziviler Helfer indes kaum, sind teilweise dogmatisch verfehlt, in ihrer Reichweite verbesserungsfähig und haben darüberhinaus in Praxis und Schrifttum kaum Resonanz gefunden. Auch angesichts aktueller Gewalteskalationen gegen Rettungskräfte im Rahmen der Blockupy-Proteste und immer neuer »Gaffer«-Fälle gilt es den Schutz von Helfern und Hilfeleistungen grundsätzlich zu überdenken.

\section{Einführung}

Mit dem 44. Gesetz zur Änderung des Strafgesetzbuches ${ }^{1}$ hat sich der Gesetzgeber neben anderen Anliegen damit befasst, wie professionelle Helfer der Feuerwehr, des Katastrophenschutzes und der Rettungsdienste bei ihren Einsätzen besser geschützt werden können. ${ }^{2}$ Das Ergebnis dieser Auseinandersetzung sind zwei Tatbestände, die den Einsatz der Rettungskräfte strafrechtlich absichern sollen: $\S 305$ a StGB soll den Schutz der Einsatzmittel, also Fahrzeuge und Gerät professioneller ziviler Helfer, durch eine Erweiterung des Tatbestands der Zerstörung wichtiger Ar-

1 44. Gesetz zur Änderung des Strafgesetzbuches - Widerstand gegen Vollstreckungsbeamte v. 1. 11. $2011 \mathrm{mWv}$ 5. 11. 2011 (BGBl. I S. 2130).

2 Für generelle Ausführungen zu den Änderungen durch das 44. StrÄndG vgl. Steinberg/Zetzmann/Dust, JR 2013, 7 ff.

*Kontaktperson: Martin Heger, der Autor ist Inhaber des Lehrstuhls für Strafrecht, Strafprozessrecht, Europäisches Strafrecht und Neuere Rechtsgeschichte an der Humboldt-Universität zu Berlin.

Michael Jahn, der Autor ist wissenschaftlicher Mitarbeiter an an der Humboldt-Universität zu Berlin. beitsmittel verbessern. § 114 Abs. 3 i.V.m. § 113 StGB soll parallel dazu den Schutz des Einsatzpersonals durch eine Erweiterung der Regeln über den Widerstand gegen Vollstreckungsbeamte ausbauen. Im folgenden Beitrag wird untersucht, ob der Gesetzgeber mit den genannten Regelungen den Schutz professioneller ziviler Helfer tatsächlich verbessert hat. Darüberhinaus wird der Frage nachgegangen, welche Schutzbereichserweiterungen im Zusammenhang mit der Behinderung von Rettungskräften sinnvoll sind, insbesondere ob es der Einführung eines "Gaffer-Tatbestands« bedarf und wie dieser sinnvoll in einen Zusammenhang mit den bestehenden Regelungen gebracht werden kann.

\section{Hintergrund der Änderungen in § 305 a und § 114 StGB}

Während Angriffe auf Polizeibeamte, beispielsweise bei Demonstrationen oder der Vollstreckung von Haftbefehlen, mit unschöner Regelmäßigkeit zum Tagesgeschäft gehören, sind vergleichbare Angriffe gegen Einsatzkräfte der Feuerwehr oder der Rettungsdienste in der öffentlichen Wahrnehmung eine Randerscheinung. Gleichwohl sind auch diese Helfer in ihrem Alltag die Anwendung von Gewalt gegen ihre Person oder das Einsatzgerät inzwischen gewohnt. Als besonders gravierender Fall gilt der Brandanschlag auf das von Vietnamesen bewohnte »Sonnenblumenhaus « in Rostock-Lichtenhagen im August 1992. Die zu den Löscharbeiten angerückte Feuerwehr wurde massiv bei ihrer Arbeit behindert, wie später nachzulesen ist: »Vor Ort kommen die Retter nicht an das brennende Haus heran. Die Brandstifter hindern die Feuerwehr über eine Stunde lang an den Löscharbeiten. Im dichten Steinhagel müssen sich die Feuerwehrmänner wieder zurückziehen. $\ll^{3}$ In ei-

3 Spiegel Online - Einestages v. 23. 8. 2007, http://www.spiegel.de/ einestages/rostock-lichtenhagen-als-der-mob-die-herrschaft-ueber 
nem anderen Fall in Hameln im Januar 2015 musste die Polizei ein Krankenhaus schützen, das von Angehörigen eines sich dort in Behandlung befindlichen Mannes belagert wurde. Während die Ärzte in der Notaufnahme um das Leben des Mannes kämpften, wurden bei der versuchten Stürmung der Klinik durch den »Familien-Clan « 14 Beamte verletzt und das Klinikgebäude erheblich beschädigt. ${ }^{4} \mathrm{Aus}$ der jüngsten Vergangenheit bleibt insbesondere auch die sog. »Blockupy-Bewegung " und das Geschehen zur Eröffnung des neuen Gebäudes der Europäischen Zentralbank (EZB) in Frankfurt am Main in Erinnerung. Demonstranten hatten in den Straßen rund um das neue Gebäude der EZB brennende Blockaden errichtet, die die ganze Frankfurter Innenstadt in einen dunklen Rauch hüllten. Die Feuerwehr, die zum Löschen der Barrikaden angerückt war, wurde bei ihren Arbeiten von einigen Demonstranten massiv behindert und vereinzelt mit Steinen beworfen, ${ }^{5}$ worauf die hessische Landesregierung prompt damit reagiert hat, einen deutlich erweiterten Schutz für Polizei- und Rettungskräfte zu fordern. ${ }^{6}$

Obgleich Fälle wie diese in der medialen Wahrnehmung bisher eine Ausnahme bleiben, haben Angriffe auf professionelle zivile Helfer in deren Berufsalltag nach Einschätzung der Fachverbände eine besorgniserregende $\mathrm{Zu}$ nahme zu verzeichnen. ${ }^{7}$ Der Verband Deutscher Feuerwehr verweist in diesem Zusammenhang beispielhaft auf eine gestiegene Fallzahl an Übergriffen auf die Mitarbeiter der Feuerwehr Hamburg. ${ }^{8}$ Insgesamt stehe die Zunahme von Angriffen gegen Rettungskräfte aber mit einem gesamtgesellschaftlichen Problem in Zusammenhang: Die Feuerwehr und andere zivile Helfer »in Uniform« würden

nahm-a-946806.html; für eine Zusammenfassung des Geschehens vgl. auch OLG Rostock NStZ 2001, $199 \mathrm{f}$.

4 Tagesspiegel v. 15.1. 2015, http://www.tagesspiegel.de/weltspie gel/ausschreitungen-vor-krankenhaus-in-hameln-verletzte-polizis ten-bei-krawallen-nach-todessturz-aus-gericht/11233292.html.

5 Spiegel Online v. 18. 3. 2015, http://www.spiegel.de/politik/deutsc hland/blockupy-protest-tausende-in-frankfurt-erwartet-a-1024115.ht $\mathrm{ml}$.

6 Vgl. den Gesetzesantrag BR-Drs. 165/15 »Initiative Schutzparagraph 112«, den die hessische Landesregierung am 14. 4. 2015 in den Bundesrat eingebracht hat und der eine massive Ausweitung des strafrechtlichen Schutzes von Polizei und Rettungskräften vorsieht.

7 Vgl. nur die Stellungnahme des Deutschen Feuerwehrverbandes zum Gesetz zur Änderung des Strafgesetzbuches - StRÄndG, u.a. Widerstand gegen Vollstreckungsbeamte, http://www.dfv.org/filead $\mathrm{min} / \mathrm{dfv} /$ Dateien/Fachwissen/Positionen/DFV-Positionspapier_Gew alt_gegen_Einsatzkraefte.pdf.

8 Stellungnahme DFV, S. 1; dort sei es demnach 2010 zu 39 Übergriffen auf Beamtinnen und Beamte der Feuerwehr gekommen, darunter Körperverletzungen und Bedrohungen mit Schuss- und Hiebwaffen. heute - insbesondere in urbanen Milieus - vermehrt als staatliche Organisationen im Sinne von »Polizei« wahrgenommen. ${ }^{9}$ In vielen Situationen richte sich aggressives Verhalten ohne genauere Betrachtung der Berufsgruppe gegen jede Form der »Staatlichkeit«. Die Differenzierung zwischen vermeintlich "guten« bzw. aus politischer Warte "neutralen« und "schlechten« Einsatzkräften habe sich damit zunehmend aufgelöst.

Davon unabhängig sind zivile Helfer aber auch dadurch gefährdet, dass sie sich häufig den Einsatzort mit Polizeikräften teilen und damit im weiteren Bereich der besonders sensiblen Eingriffsverwaltung tätig werden, der speziell als konfliktträchtig gilt. ${ }^{10}$ Das betrifft beispielsweise Rettungssanitäter, die im Zusammenhang mit Ausschreitungen bei Demonstrationen ihren Dienst tun, oder Feuerwehrkräfte, die bei einem Polizeieinsatz unterstützend tätig werden. Aggressionen am Einsatzort richten sich dabei nicht nur gegen die Einsatzkräfte in Person. Ziele sind häufig auch die dort verwendeten Einsatzmittel, wie z.B. Rettungswagen, Funktechnik und sonstige Notfallausrüstung. Der Gesetzgeber hat sich in der Gesetzesbegründung zum 44. SträndG dieser Einschätzung angeschlossen und begründet die Erweiterung des Schutzbereichs ebenfalls mit der »deutlichen Zunahme an Übergriffen auf Einsatzkräfte der Feuerwehr und der Rettungsdienste «. ${ }^{11}$

\section{Auswirkungen der Änderungen in § 305 a und § 114 StGB}

\section{Der Schutz der Einsatzmittel durch § 305 a StGB}

$\S 305$ a stellt die Zerstörung wichtiger Arbeitsmittel unter Strafe. Bis zum 5. 11. 2011 waren ausschließlich technische Arbeitsmittel lebenswichtiger Betriebe, die der öffentlichen Versorgung dienen ( $\S 316 \mathrm{~b} \mathrm{StGB})$, sowie Kraftfahrzeuge der Polizei und der Bundeswehr durch diese Norm geschützt. Rettungswagen, Fahrzeuge und Gerät der Feuerwehr oder des Technischen Hilfswerks konnten zu diesem Zeitpunkt allein durch den Tatbestand der einfachen Sachbeschädigung gem. § 303 StGB und gegebenenfalls durch eine gemeinschädliche Sachbeschädigung gem.

9 Ganz im Gegenteil dazu arbeiten viele Feuerwehren in ehrenamtlicher Tradition, Stellungnahme DFV, S. 2.

10 Stellungnahme DFV, S. 4.

11 BT-Drs. 17/4143, S. 7; ebenso die hessische Landesregierung in BR-Drs. 165/15. 
$\S 304$ StGB vor Beeinträchtigungen geschützt werden. ${ }^{12}$ Über eine Erweiterung in $\S 305 a$ Abs. 1 Nr. 3 StGB wurde der Schutzbereich dieses besonderen Sachbeschädigungsdelikts nun auf Kraftfahrzeuge der genannten zivilen Helfer, also alle Einsatzwagen der Rettungsdienste oder etwa Löschfahrzeuge der Feuerwehr, erweitert. § 305a Abs. 1 Nr. 2 erfasst neben den Fahrzeugen außerdem die technische Ausrüstung der Einsatzkräfte, sofern diese für den Einsatz wesentlich und von bedeutendem Wert ist. ${ }^{13}$ Der Strafrahmen des $\S 305$ a StGB sieht bis zu fünf Jahre Freiheitsstrafe vor und erhöht den Schutz gegenüber der bisherigen Regelung deutlich, nach der solche Handlungen von $\S 303$ StGB erfasst wurden, der lediglich eine Freiheitsstrafe von bis zu zwei Jahre vorsieht. Die Änderung sendet auch eine nicht zu unterschätzende Signalwirkung aus: $\S 305$ a StGB schützt nämlich nicht primär das Eigentum im Einzelnen, sondern das Allgemeininteresse an der Funktionstüchtigkeit lebensnotwendiger Infrastruktureinrichtungen (vgl. den Verweis auf $\S 316 \mathrm{~b} \mathrm{StGB}),{ }^{14}$ was auch den hohen Strafrahmen erklärt. Die Aufnahme in diesen »elitären Kreis« ist daher besonders bemerkenswert und letztlich auch insofern überfällig, als Polizei und Bundeswehr schon bisher Schutzadressaten des § 305a Abs. 1 Nr. 2 und 3 StGB waren. Besondere Relevanz erhält § 305a StGB außerdem dadurch, dass der Tatbestand eine Katalogtat des §129a II StGB bildet und damit bei dem Verdacht auf eine solche Straftat besondere Ermittlungsbefugnisse, wie beispielsweise die akustische Wohnraumüberwachung gem. § 100c StPO oder die Durchsuchung nichtverdächtiger Personen nach $\S 103$ StPO, legitimiert. ${ }^{15}$

\section{Der Schutz des Einsatzpersonals durch $\S 114$ Abs. 3 StGB}

Neben der Änderung in $\S 305$ a StGB sollte durch das 44. StrÄndG der Schutz professioneller ziviler Helfer durch die Erweiterung des $\S 114$ StGB um einen dritten Absatz gestärkt werden. Dieser bestimmt, dass bestraft wird, wer bei Unglücksfällen oder gemeiner Gefahr oder Not Hilfeleis-

12 Als »Gegenstand des öffentlichen Nutzens « und damit als taugliches Tatobjekt des $\S 304$ Abs. 1 StGB wurden insofern bisher Krankenund Krankentransportwagen gewertet (OLG Düsseldorf, NJW 1986, 2123; aber offen gelassen durch BGH NJW 1983, 1437).

13 Ein Großteil der Ausrüstung der Feuerwehr unterscheide sich laut DFV sowohl taktisch als auch betriebswirtschaftlich kaum noch von Kraftfahrzeugen, Stellungnahme DFV, S. 4.

14 Vgl. Heger, in: Lackner/Kühl, StGB, § 305a Rn. 1.

15 Fischer, StGB, § 305a Rn. 2a; vgl. zu weiteren Ermittlungsbefugnissen durch §§ 129a II i.V.m. § 305a StGB Schäfer, in: MK-StGB, $\S 129$ a Rn. 88. tende der Feuerwehr, des Katastrophenschutzes oder eines Rettungsdienstes durch Gewalt oder durch Drohung mit Gewalt behindert oder sie dabei tätlich angreift. Die Norm verweist dazu auf die Rechtsfolge des $\S 113$ StGB und stellt damit alle Personen, die als Feuerwehrleute, Katastrophenhelfer oder Rettungssanitäter und Notärzte tätig sind, unter denselben Schutz, den auch Vollstreckungsbeamte nach $\S 113$ StGB genießen.

\section{a) Geschützter Personenkreis des $\S 114$ Abs. 3 StGB und das Rechtsgutsproblem}

Einbezogen in den erweiterten Schutzbereich der Norm sind »Hilfeleistende der Feuerwehr, des Katastrophenschutzes oder eines Rettungsdienstes«. Dabei soll dies umfassend gelten, ${ }^{16}$ das heißt, die Formulierung »eines « Rettungsdienstes ist keineswegs technisch gemeint, sondern umfasst neben den Berufsfeuerwehren zum Beispiel auch Helfer der Freiwilligen Feuerwehren. ${ }^{17}$ Geschütztes Rechtsgut der $\S \S 113,114$ StGB ist hingegen die rechtmäßige Betätigung des Staatswillens. ${ }^{18}$ Die Normen sollen also staatliche Vollstreckungsakte und das Gewaltmonopol des Staates verteidigen, weshalb es für $\S 113$ StGB entscheidend auf den Begriff des Vollstreckungsbeamten ankommt und $\S 114$ StGB noch ausweislich der amtlichen Überschrift nur auf solche Personen Anwendung finden soll, die Vollstreckungsbeamten gleichstehen. $§ 114$ StGB befindet sich selbst im 6. Abschnitt des Besonderen Teils, der mit »Widerstand gegen die Staatsgewalt« überschrieben ist. Hilfeleistende der Feuerwehr, des Katastrophenschutzes oder der Rettungsdienste sind jedoch erstens nicht zwingend staatliche Akteure, sondern - wie erwähnt - häufig als private Zivilschutzeinrichtungen oder freiwillige Feuerwehren organisiert. ${ }^{19}$ Und sie führen zweitens, und das ist entscheidend, keine Vollstreckungshandlungen im Sinne des $\S 113$ StGB aus. Die Einordnung des Schutzes ziviler Helfer im Bereich der $\S \S 113,114$ StGB stellt damit einen Fremdkörper dar. ${ }^{20}$ Es bleibt insgesamt rätselhaft, warum der Schutz ziviler Helfer zwingend über eine Gleich-

16 Vom Schutzbereich umfasst sind "sämtliche Rettungsdienste«, BT-Drs. 17/6505, S. 5.

17 Bosch, in: MK-StGB, § 114 Rn. 10; vgl. auch Wolters, in: SK-StGB, $\S 114$ Rn. 10.

18 Heger, in: Lackner/Kühl, StGB, § 113 Rn. 1. 19 Heger, in: Lackner/Kühl, StGB, § 114 Rn. 4.

20 Zopfs, GA 2012, 259ff., 264; vgl. auch Steinberg/Zetzmann/Dust, JR 2013, 7 ff., 8: »teleologisch systemwidrig«; Singelnstein/Puschke, NJW 2011, 3473 ff., 3473: »unsystematisch und wenig durchdacht«. 
stellung mit Vollstreckungsbeamten erreicht werden musste..$^{21}$

Da das geschützte Rechtsgut der §§ 113, 114 StGB auf den dritten Absatz des $\S 114$ StGB nicht recht passt, ist zu überlegen, welche Schutzfunktion der neue Tatbestand übernehmen soll: Die individualschützende Funktion des $\S 114$ Abs. 3 StGB erscheint dabei als das leitende Motiv. Geschützt wären danach primär die Willens- und Betätigungsfreiheit sowie die körperliche Unversehrtheit der zivilen Helfer. ${ }^{22}$ Die Norm schützt jedoch ferner auch die Hilfeleistungshandlungen selbst und deren ungestörte Durchführung, damit Rettungschancen gewahrt werden. Mittelbar geschützt sind damit letztlich die Individualrechtsgüter, die körperliche Unversehrtheit und das Leben, der bei Unglücksfällen oder gemeiner Gefahr oder Not Betroffenen. ${ }^{23}$ Von dieser Prämisse ausgehend wird noch einmal deutlich, dass die Einordnung des Schutzes ziviler Helfer in $§ 114$ StGB systematisch wenig Sinn macht. Überzeugender wäre es gewesen, diesen Schutz im weiteren Kontext des $\S 323$ c StGB zu regeln, der bereits viele der tatbestandlichen Merkmale enthält, die auch $\S 114$ Abs. 3 StGB aufweist. ${ }^{24}$

\section{b) Einschränkung der möglichen Tatbeteiligten bei $\S 114$ Abs. 3 StGB}

Bei der Konzeption des $\S 114$ Abs. 3 StGB hatte der Gesetzgeber augenscheinlich Situationen im Sinn, in denen Dritte die Hilfeleistung bei einem Verletzten behindern. So zum Beispiel der Einsatz von Feuerwehrkräften in einem Mehrfamilienhaus, bei dem die Einsatzkräfte von Mitgliedern einer dort wohnhaften Familie behindert und tätlich angegriffen werden, oder der Rettungssanitäter, der einem Bewusstlosen auf einem Volksfest helfend plötzlich von Schaulustigen, Freunden oder Verwandten des Hilfsbedürftigen angegangen wird. Ein Großteil der praxisrelevanten Fälle könnte indes insofern anders liegen, als der Angreifer sogleich selbst der Verletzte ist. So zum Beispiel der alkoholisierte und bewusstlose Patient, der im Rettungswagen plötzlich aufwacht und den Rettungssanitäter tätlich angreift oder der unter Drogen stehende Patient, der den Arzt am Unfallort in die Hand beißt. Die Anwendbarkeit des $\S 114$ Abs. 3 StGB ist in diesen Fällen auch nicht

21 Vgl. auch Paeffgen, in: Kindhäuser/Neumann/Paeffgen StGB, $\S 114$ Rn. 11a: "materiell geradezu abwegige Anbindung an $\S 113$ $\mathrm{StGB} \ll$.

22 So auch Singelnstein/Puschke, NJW 2011, 3473 ff., 3475.

23 So ebenfalls Singelnstein/Puschke, NJW 2011, 3473 ff., 3475.

24 S.u. VI. per se dadurch ausgeschlossen, dass ein Patient noch in der Lage ist, sich tätlich gegen seinen Helfer zu wehren. Dies allein schließt nicht zwingend das Vorliegen eines Unglücksfalls aus. Denn trotz aktiver Gegenwehr kann es sein, dass der Patient dennoch auf Hilfe angewiesen ist. Für einen Unglücksfall kommt es schließlich allein darauf an, dass bei Abbruch der Hilfeleistungshandlungen dem Verletzten weiterhin erhebliche Gefahren drohen..$^{25}$

Unter dem Aspekt, dass § 114 Abs. 3 StGB neben den Rechtsgütern der Helfer in Abgrenzung zu $\S 240$ StGB die Hilfeleistung an sich und damit mittelbar die Individualrechtsgüter des Hilfsbedürftigen schützt, scheint es in diesen Fällen angemessen, Behinderungen und tätliche Angriffe durch den Hilfsbedürftigen selbst vom Tatbestand auszunehmen. Hilfsbedürftige können im Rahmen der Patientenautonomie auf eine Rettung verzichten und unterliegen grundsätzlich keiner Pflicht, die Behandlung eines Arztes oder Rettungssanitäters über sich ergehen zu lassen. ${ }^{26}$ Die Hilfeleistung und damit ebenso die Individualrechtsgüter der Hilfsbedürftigen sind damit in dieser Fallsituation nicht schutzwürdig. Berücksichtigt man dies, so bleibt in den Fällen der Identität von Hilfsbedürftigem und Täter des § 114 Abs. 3 StGB nur noch das Unrecht der Körperverletzung oder Nötigung übrig. Da § 114 StGB nach seinem Wortlaut auch einen Angriff durch den Hilfsbedürftigen selbst umfasst, muss der Tatbestand in diesen Fällen teleologisch reduziert werden. ${ }^{27}$

\section{c) Tatbestandsmäßige Situation des § 114 Abs. 3 StGB}

$\S 114$ Abs. 3 StGB setzt als tatbestandsmäßige Situation das Vorliegen eines Unglücksfalls oder einer gemeinen Gefahr oder Not sowie als Tatobjekt Hilfeleistende aus den oben benannten Personengruppen voraus. Die tatbestandsmäßige Situation erinnert damit stark an die Voraussetzungen des Tatbestands der Unterlassenen Hilfeleistung nach $\S 323 \mathrm{c}$ StGB und grenzt sich von $\S 114$ Abs. 1 und 2 StGB dadurch ab, dass das Erfordernis einer Vollstreckungshandlung durch die Hilfeleistungssituation substituiert wird. Während man für die Begriffe »Unglücksfall« und "gemeine Gefahr und Not« auf die vorhandene Dogmatik zu $\S 323$ c StGB zurückgreifen kann, ${ }^{28}$ stellt sich jedoch die schwieriger $\mathrm{zu}$ beantwortende Frage, wie der Begriff »Hilfeleistende« jenseits der Frage der Zugehörig-

25 Kühl, in: Lackner/Kühl, StGB, § 323c Rn. 2. 26 Eser, in: Schönke/Schröder StGB, § 223 Rn. 37. 27 Eine Bestrafung nach den $\S \S 223 \mathrm{ff}$., 240 StGB bleibt daneben weiterhin möglich; siehe auch unten III. 2. d. 28 Vgl. Kühl, in: Lackner/Kühl, StGB, § 323c Rn. 2f. m.w. N. 
keit $\mathrm{zu}$ einer der genannten Personengruppen auszulegen ist. § 323c StGB konkretisiert den artverwandten Begriff der »Hilfeleistung « als nur dann tatbestandsmäßig, wenn diese erforderlich und zumutbar ist. Eine unterlassene Hilfeleistung, die gleichwohl nicht erforderlich ist oder sich in der Situation als unzumutbar herausgestellt hat, ist damit nicht tatbestandsmäßig. Dies betrifft z.B. den Fall, dass ein Verunglückter wider Erwarten noch selbst in der Lage ist, sich ausreichend zu helfen und daher auf fremde Hilfe nicht angewiesen ist oder diesem bereits von anderer Seite Hilfe geleistet wird. ${ }^{29}$ Das passive Verhalten eines potentiellen Retters bleibt dann im Rahmen des § 323c StGB tatbestandslos, da seine Hilfeleistung nicht erforderlich ist.

Der Wortlaut des § 114 Abs. 3 StGB verlangt allein mit dem Begriff »Hilfeleistende« nicht ausdrücklich, dass der Beitrag der Hilfeleistung ein Akt der Notwendigkeit und mithin erforderlich sein muss. Aus der systematischen Ähnlichkeit zu § 323c StGB wird dies aber teilweise in die Norm mithineingelesen. Wolters verlangt beispielsweise, dass »die Handlung der Feuerwehr etc. zur Abwendung einer Gefahr geeignet ist und sie die Wahrscheinlichkeit des Eintritts eines (weiteren) Schadens verringert ${ }^{30}{ }^{30} \mathrm{Ge}-$ stützt wird diese Ansicht darauf, dass § 114 Abs. 3 StGB unter Berufung auf das Behindern als Tathandlung als Erfolgsdelikt eingestuft wird. ${ }^{31}$ Die Logik dieses Arguments verläuft dabei scheinbar folgendermaßen: Geht es um ein Erfolgsdelikt, so müsse die Hilfeleistung der Rettungskräfte tatsächlich behindert worden sein. Eine Hilfeleistung könne aber nur dann behindert werden, wenn sie auch erforderlich ist. Denn eine nicht erforderliche Hilfeleistung ist eigentlich keine echte Hilfeleistung, sondern nur ein vergebliches Bemühen. Die Verhinderung dieses Bemühens ist dann nicht mehr als ein strafloser (untauglicher) Versuch des § 114 Abs. 3 StGB.

Ob eine Hilfeleistung tatsächlich erforderlich ist oder nicht, wird in vielen Fällen jedoch gerade bei Handlungen im Vorfeld der eigentlichen Rettungsmaßnahme schwer festzustellen sein, wie beispielsweise bei der Anfahrt der Helfer zum Unglücksort. ${ }^{32}$ Hier kann sich in einigen Fällen herausstellen, dass der Einsatz eines weiteren Rettungswagens oder Löschfahrzeugs der Feuerwehr zur Abwendung eines Unglücks nicht mehr notwen-

29 Sternberg-Lieben/Hecker, in: Schönke/Schröder StGB, §323c Rn. 15.

30 Wolters, in: SK-StGB, § 114 Rn. 11.

31 Singelnstein/Puschke, NJW 2011, 3473ff., 3474; Bosch, in: MKStGB, § 114 Rn. 11; Dallmeyer, in: BeckOK StGB, § 114 Rn. 4; Wolters, in: SK-StGB, § 114 Rn. 11.

32 Fischer, StGB, § 114 Rn. 8. dig war, da bereits ausreichend Personal und Arbeitsmittel vor Ort waren.

Gegen eine eingeschränkte Auslegung des Begriffs »Hilfeleistende« spricht auch, dass sich eine solche nicht ohne weiteres mit Blick auf die 2. Handlungsvariante des tätlichen Angriffs erschließt. Geht man nämlich davon aus, dass die Erforderlichkeit der Hilfeleistung sich gedanklich erst in Verbindung mit dem Merkmal der 1. Handlungsvariante "Behindern" entwickelt, stellt sich die Frage, ob es für das tätliche Angreifen eines Helfers nach der 2. Handlungsvariante ebenso auf die Erforderlichkeit seiner Hilfeleistung ankommt. Um die Kohärenz des Tatbestands zu wahren, dürfte auch diese Handlungsvariante die Retter nur schützen, sofern deren Hilfeleistungen erforderlich sind. Dies mutet gerade deshalb paradox an, als § 114 Abs. 3 StGB primär die Rechtsgüter der Retter und nicht der zu rettenden Personen in den Blick nimmt. Für den Schutz der körperlichen Unversehrtheit, den die Variante des tätlichen Angriffs in den Blick nimmt, kann es keinen Unterschied machen, ob die durch den Retter intendierte Hilfeleistung erforderlich oder entbehrlich ist.

$\mathrm{Zu}$ wenig Berücksichtigung findet außerdem die Tatsache, dass der Wortlaut des Gesetzes gar keine Behinderung der Hilfeleistung, sondern eine Behinderung der Hilfeleistenden verlangt, die Hilfeleistung also gar nicht als eigenständiges Merkmal ausgestaltet ist. ${ }^{33}$ Dies lässt darauf schließen, dass der Begriff $»$ Hilfeleistende« weiter verstanden werden muss als der Begriff "Hilfeleistung " bei $\S 323$ c StGB. Es genügt für $\S 114$ Abs. 3 StGB daher bereits, wenn der Täter eine Handlung der zivilen Helfer behindert, die auf die Hilfeleistung bei einem Unglücksfall ausgerichtet war. ${ }^{34}$ Ob diese Handlung zur Verhinderung weiteren Schadens beim Hilfsbedürftigen tatsächlich erforderlich war, spielt für die Erfüllung des Tatbestandes keine Rolle. Es kommt also nicht auf einen weitergehenden Erfolg im Sinne einer tatsächlichen Behinderung einer Hilfeleistung, also der Verschlechterung von Rettungschancen, an.

\section{d) Das Verhältnis von § 114 Abs. 3 StGB zu § 240 StGB}

Ob die neue Regelung in $\S 114$ Abs. 3 StGB zivile Retter tatsächlich besser zu schützen vermag, hängt außerdem davon ab, wie das Verhältnis zu konkurrierenden Normen wie den $\S \S 223 \mathrm{ff}$. und $\S 240$ StGB ausgestaltet ist. Während

33 Vgl. auch Zopfs, GA 2012, 259 ff., 274.

34 Fischer, StGB, § 114 Rn. 8. 
der Täter des $\S 114$ Abs. 3 StGB in Tateinheit wegen Körperverletzungsdelikten bestraft werden kann, ist das Verhältnis des Tatbestands zur Nötigung ungleich komplizierter, was auch durch die bisherige Einordnung des $\S 113$ StGB zur Nötigung beeinflusst ist. Vor dem 44. StrÄndG war das Verhältnis von $\S 113$ StGB zu § 240 StGB durch eine Privilegierung des Angriffs auf Vollstreckungsbeamte geprägt. ${ }^{35} \S 113$ StGB galt als lex specialis gegenüber der einfachen Nötigung und sperrte deren Anwendung im Rahmen der Gesetzeskonkurrenz. ${ }^{36}$ Der höhere Strafrahmen des $\S 240$ StGB kam damit für den Täter nicht mehr in Betracht. Die Tat konnte nur noch mit der niedrigeren Strafe des § 113 StGB geahndet werden. Der Grundsatz der Spezialität liegt immer dann vor, wenn ein Tatbestand mit allen Merkmalen vollständig in einem anderen Tatbestand enthalten ist, und letzterer noch mindestens eine weitere konkretisierende Tatbestandsvoraussetzung aufweist. ${ }^{37}$ Die Einordnung des Verhältnisses von $\S 113$ StGB zu § 240 StGB im Rahmen der Spezialität führte auch bislang schon zu Problemen, da die klassische Definition nicht eindeutig passte. So bringt zwar $\S 113$ StGB mit der Vornahme einer Vollstreckungshandlung eine weitere Tatbestandsvoraussetzung mit, enthält jedoch nicht alle Tatbestandsalternativen des $\S 240$ StGB. Schließlich fordert $\S 113$ StGB als Nötigungsmittel »Gewalt oder die Drohung mit Gewalt«. Das weniger einschneidende Nötigungsmittel der Drohung mit einem empfindlichen Übel in $\S 240$ StGB wird von $\S 113$ StGB hingegen nicht übernommen, was für diese Konstellation schon bisher zu Auseinandersetzungen führte. ${ }^{38} \mathrm{Die}$ Feststellung, dass § 240 StGB durch Spezialität gesperrt ist, bedurfte daher weitergehend einer teleologisch-systematischen Auslegung der Vorschriften. ${ }^{39}$ Ausgangspunkt und Hauptargument für die genannte Einordnung war die geringere Strafdrohung des $\S 113$ StGB gegenüber der Nötigung. ${ }^{40}$ Diese wurde mit der Annahme einer besonderen psychischen Situation auf Seiten des Täters gerechtfertigt. Der Bürger stehe dem Staat bei dem Vollzug von Vollstreckungshandlungen in einer besonders konfliktträchtigen und konfrontativen Sondersituation gegenüber und das

35 Vgl. zur überkommenen Privilegierungsthese Eser, in: Schönke/ Schröder StGB, § 113 Rn. 3.

36 Eser, in: Schönke/Schröder StGB, § 113 Rn. 68.

37 Kühl, Strafrecht AT, § 21 Rn. 52.

38 Für einen generellen Ausschluss des $\S 240$ StGB auch bei Drohungen mit einem empfindlichen Übel: Horn/Wolters, in: SK-StGB, § 113 Rn. 23; Eser, in: Schönke/Schröder StGB, § 113 Rn. 68 m.w. N.; anders hingegen Rengier Strafrecht BT II, § 54 Rn. 28; Heger, in: Lackner/ Kühl, StGB, § 113 Rn. 26 m.w.N.

39 Kühl, Strafrecht AT, § 21 Rn. 52.

40 BGHSt 48, 233;
Strafrecht sollte dem Täter daher mit Nachsicht begegnen. ${ }^{41}$

Mit den Änderungen vom 5. 11. 2011 ist zumindest die rechtsfolgenbegründete Privilegierung weggefallen, da der Strafrahmen des $§ 113$ StGB nun auch eine Freiheitsstrafe bis zu drei Jahren und damit dieselbe Rechtsfolge wie die einfache Nötigung vorsieht. Das Verhältnis von $\S 113$ StGB zu § 240 StGB wird ungeachtet dessen nach allgemeiner Ansicht weiterhin durch den Grundsatz der Spezialität bestimmt. ${ }^{42}$ Dies ist auch sinnvoll unter Berücksichtigung der oben genannten Hintergründe, da der Täter so immer noch auf der Ebene der Nötigung mit einem empfindlichen Übel und den Irrtumsregelungen des $\S 113$ Abs. 3 und 4 StGB privilegiert wird und diese Privilegierung weiterhin ihre Legitimation durch die besondere psychische Belastungssituation im Rahmen des § 113 StGB erfährt. ${ }^{43}$

Vor dem Hintergrund dieser Privilegierungsproblematik stellt sich jedoch die Frage, wie § 114 Abs. 3 StGB und damit der Schutz der professionellen zivilen Helfer im Verhältnis zur Nötigung einzuordnen ist; denn hier passt die ursprüngliche Privilegierungsbegründung mangels Vollstreckungshandlung von vornherein nicht. ${ }^{44}$ Der Täter sieht sich eben nicht einer Diensthandlung eines Vollstreckungsbeamten gegenüber und befindet sich damit nicht in der oben beschriebenen Sondersituation. Dennoch wird weit überwiegend kein Unterschied zu dem Verhältnis von $\S 113$ StGB zu $\S 240$ StGB gemacht. ${ }^{45}$ Dass $\S 114$ Abs. 3 StGB lex specialis zu $§ 240$ StGB sein soll, wird mit der systematischen Verbundenheit zu § 113 StGB begründet sowie mit dem speziellen Anwendungsbereich, den die Norm allein für Angriffe auf professionelle Helfer schaffe. ${ }^{46}$ Auch durch die zusätzliche Schutzrichtung in Abs. 3, die neben der körperlichen Unversehrtheit und Willensfreiheit der Helfer auch die Individualrechtsgüter der Hilfeadressaten umfasst, soll der Charakter als Spezialnorm deutlich wer-

41 Bosch, in: Münchner Kommentar zum StGB, § 113 StGB Rn. 1.; ein knapper Überblick zum Hintergrund der Norm und ihrer Entwicklung seit 1870 bei Steinberg/Zetzmann/Dust, JR 2013, 7 ff., 9 f.

42 Eser, in: Schönke/Schröder StGB, § 113 Rn. 68 m.w.N.

43 Kritisch zur Theorie der besonderen psychischen Belastungssituation Steinberg/Zetzmann/Dust, JR 2013, 7 ff., 10; Bosch, JURA 2011, 268ff., 268; Paeffgen, in: Kindhäuser/Neumann/Paeffgen StGB, § 113 Rn. 3.

44 Steinberg/Zetzmann/Dust, JR 2013, 7 ff., 8.

45 Vgl. nur Fahl, ZStW 2012, 311ff., 323, der darin sogar den Grund sieht, warum der Gesetzgeber bewusst den Tatbestand im Zusammenhang mit § 113 StGB geregelt hat; außerdem Singelnstein/Puschke, NJW 2011, 3473ff., 3475; Paeffgen, in: Kindhäuser/Neumann/Paeffgen StGB, § 114 Rn. 13; Eser, in: Schönke/Schröder StGB, § 114 Rn. 25. 46 Singelnstein/Puschke, NJW 2011, $3473 \mathrm{ff} ., 3475$. 
den. ${ }^{47}$ Überzeugender scheint es jedoch, für das Verhältnis von $\S 114$ Abs. 3 zu § 240 StGB Tateinheit anzunehmen. Sonst würde ein Festhalten an der bisherigen Auslegung im Ergebnis eine Benachteiligung ziviler Helfer im Vergleich zur Rechtslage vor dem 44. StrÄndG darstellen. Wenn nämlich die Anwendung von § 240 StGB durch § 114 Abs. 3 StGB gesperrt ist, sind solche Handlungen gegenüber Hilfeleistenden ausgenommen, die nur die Androhung eines empfindlichen Übels umfassen. ${ }^{48} \S 114$ Abs. 3 StGB setzt schließlich wie der Widerstand gegen Vollstreckungsbeamte voraus, dass der Täter seine Behinderungen durch » Gewalt oder durch Drohung mit Gewalt« umsetzt.

Dieses dem Gesetzeszweck zuwiderlaufende Ergebnis kann allenfalls mit Unbedacht bei der Gesetzgebung erklärt werden. ${ }^{49}$ Denn ausweislich der Gesetzesbegründung heißt es, die neue Regelung in $\S 114$ Abs. 3 StGB solle »unabhängig von bereits vorhandenen Sanktionsmöglichkeiten " Feuerwehrleute und Rettungskräfte schützen. ${ }^{50}$ Aus diesem Grund kann $\S 114$ Abs. 3 StGB nicht als Spezialvorschrift mit Sperrwirkung gegenüber § 240 StGB interpretiert werden..$^{51}$ Es liegt vielmehr der Schluss nahe, dass das mehrheitliche Festhalten am Grundsatz der Spezialität auch für $\S 114$ Abs. 3 StGB eine reflexhafte Handlung ist, die allein durch die dogmatisch verfehlte Einordnung der Norm im Bereich der $\S \S 113,114$ StGB geleitet ist.

$\S 114$ Abs. 3 StGB sollte vielmehr als eine von Rechtsgut und Systematik der §§ 113, 114 Abs. 1 und 2 StGB getrennt aufzufassende Regelung verstanden werden, die $\S 240$ StGB nicht verdrängt, sondern zusätzlichen Schutz entfaltet.

\section{e) Zwischenergebnis}

Die Änderungen durch das 44. StrÄndG können den Schutz ziviler professioneller Helfer nicht entscheidend verbessern. Zwar erhöht § 305a StGB den Schutz der Einsatzmittel, die Erweiterung in $\S 114$ Abs. 3 StGB ist jedoch in mehrfacher Hinsicht problematisch: Zum einen geht der

47 Singelnstein/Puschke, NJW 2011, 3473 ff., 3475.

48 Das können z.B. gegenstandslose Strafanzeigen oder Dienstaufsichtsbeschwerden sein, mit denen Rettern gedroht wird.

49 Bosch, JURA 2011, 268ff., 270; vgl. auch Zopfs, GA 2012, 259ff., 265: »[...] lassen die Entwurfsbegründungen und die Stellungnahmen der Abgeordneten erkennen, dass die Voraussetzungen der Norm und das systematische Verhältnis zu anderen Vorschriften vielfach unbekannt sind.«.

50 BT-Drucks. 646/10, S. 6.

51 So auch Bosch, in: MK-StGB, § 114 Rn. 11; Zopfs, GA 2012, 259 ff., $274 f$.
Strafrahmen des $\S 114$ Abs. 3 StGB nicht über das hinaus, was auch bisher über $\S \S 223 \mathrm{ff} ., 240 \mathrm{StGB}$ abgedeckt war. Zum anderen stellt die Einordnung bei den $\S \S 113,114$ StGB die Anwendbarkeit der Nötigung gem. § 240 StGB in den Fällen der Behinderung von Hilfeleistungen in Frage, was im äußersten Fall zu einem abgesenkten Schutzniveau führt. Außerdem bestehen Auslegungsprobleme, da der Tatbestand auf vorhandene Merkmale der Unterlassenen Hilfeleistung aufbaut, diese mit dem Merkmal »Hilfeleistende« jedoch in neuer Form verwendet.

\section{Ein »Gaffer-Tatbestand« als weitere Stufe des Schutzes von Rettungsbemühungen}

Die Behinderung von Feuerwehr und Rettungsdiensten erfolgt nicht selten durch eine Gruppe von Personen, die in der Literatur unter den negativ konnotierten Begriffen "Gaffer « oder "Schaulustige" geführt wird. ${ }^{52}$ Gebräuchlich ist für den gesamten die Hilfeleistung behindernden Vorgang auch die Bezeichnung "Katastrophentourismus «. ${ }^{53}$ Bezeichnend für das sog. »Gaffen « ist im Gegensatz zu den vorangegangen Beispielen, dass es den Tätern auf die Behinderung der Hilfeleistung nicht im Sinne von Absicht "gerade ankommt«, sondern eine Behinderung allenfalls mit bedingtem Vorsatz erfolgt. Die Behinderung ist in der Regel eine Nebenfolge der Befriedigung voyeuristischer Interessen und entsteht häufig auch erst durch das ungewollte Zusammenwirken einer Mehrzahl von Personen.

Der "Katastrophentourismus« als typische Variante der Behinderung von Rettungskräften am Unglücksort kann verschiedene Auswirkungen haben: Ärzte müssen sich so beispielsweise erst ihren Weg durch die Menge bahnen und können den Verletzten nicht schnell genug erreichen oder Rettungshubschrauber finden keinen geeigneten Platz, um möglichst nah am Unfallort zu landen. Die bloße körperliche Anwesenheit der Schaulustigen führt hier dazu, dass Rettungschancen verhindert oder beeinträchtigt sind. Das Verhalten Schaulustiger kann über die Verhinderung von Rettungsbemühungen hinaus auch selbst zur Gefahr werden: Um einen typischen Fall handelt es sich, wenn bei Unfällen im Straßenverkehr Autos auf der Gegenfahrbahn mit reduzierter Geschwindigkeit die Unfallstelle passieren, und dadurch selbst zu

52 Vgl. z. B. Scheffler NJW 1995, $232 \mathrm{ff}$.

53 Vgl. Schwind, Alle Gaffen - keiner hilft: Unterlassene Hilfeleistung bei Unfällen und Straftaten, 1998, S. 163. 
einem Unfallrisiko werden. Eine solche Situation ereignete sich im März 2015 auf der A57 bei Köln. Dabei haben Autofahrer mit ihren Handys einen Unfall gefilmt und fotografiert und damit für einen Stau auf der Gegenfahrbahn gesorgt, was für den Vorsitzenden der Deutschen Polizeigewerkschaft in Nordrhein-Westfalen der Auslöser war, einen gesonderten "Straftatbestand des Gaffens « zu fordern. ${ }^{54}$ Eine Forderung, die alle paar Jahre wieder auftaucht, die aber bisher kein offenes Ohr in der Politik gefunden hat.

Hierbei ist zunächst zu überlegen, ob nicht das geltende Recht eine effektive Bekämpfung des Phänomens »Gaffen« zulässt, bevor Überlegungen angestellt werden sollen, wie ein solcher Tatbestand aussehen und sich in die geltende Systematik des Strafrechts einordnen kann. ${ }^{55}$ Eine Strafbarkeit nach $\S 114$ Abs. 3 StGB scheidet für das Verhalten Schaulustiger aus. Das bloße Behindern bzw. Verhindern der Rettungsleistung allein genügt nicht. Dieses muss durch Gewalt oder durch Drohung mit Gewalt bzw. durch einen tätlichen Angriff erfolgen. Das typische Verhalten Schaulustiger ist jedoch eine Handlung, die lediglich in körperlicher Anwesenheit besteht und damit den Drohungs- und Gewaltbegriff nicht ausfüllen kann. ${ }^{56}$ In einzelnen Fällen ist allerdings eine Strafbarkeit wegen Körperverletzungs- oder Tötungsdelikten unter dem Gesichtspunkt des Abbruchs rettender Kausalverläufe denkbar. ${ }^{57}$ Allerdings wird es fast immer an der subjektiven Tatseite der $\S \S 223 \mathrm{ff}$. und $\S \S 211 \mathrm{f}$. StGB mangeln. Und auch die Strafbarkeit aus einem Fahrlässigkeitsdelikt scheitert in der Regel daran, dass der Nachweis der Kausalität nicht erbracht werden kann. ${ }^{58}$ Es bleibt schließlich eine mögliche Strafbarkeit wegen Unterlassener Hilfeleistung nach $\S 323 c$ StGB. Die tatbestandsmäßige Situation der Norm, ein Unglücksfall, eine gemeine Gefahr oder Not, ist es ja

54 Rheinische Post Online, http://www.rp-online.de/nrw/panorama /polizei-will-gaffer-haerter-bestrafen-aid-1.4943299; als Ordnungswidrigkeit gem. §§ 3 Abs. 2, 49 Abs. 1 Nr. 3 StVO i.V.m. § 24 StVG kann das verlangsamte Fahren indes verfolgt werden, wie in diesem Fall geschehen.

55 Vgl. zu den nichtstrafrechtlichen Sanktionsmöglichkeiten des "Gaffens« Scheffler NJW 1995, 232, 233f.; neben straf- und ordnungsrechtlichen Sanktionen bieten die Polizeigesetze der Länder als unmittelbare Reaktion auf Behinderungen durch «Gaffer« die Möglichkeit, einen Platzverweis auszusprechen, was in einigen Polizeigesetzen auch explizit für diesen besonderen Fall geregelt ist (vgl. z. B. ASOG Bln § 29 Abs. 1S. 2: » Die Platzverweisung kann ferner gegen eine Person angeordnet werden, die den Einsatz der Polizei, der Feuerwehr oder von Hilfs- oder Rettungsdiensten behindert.«).

56 Vgl. zum Gewaltbegriff Heger, in: Lackner/Kühl, StGB, § 240 Rn. $5 \mathrm{ff}$.

57 Scheffler NJW 1995, 232, 234.

58 Scheffler NJW 1995, 232, 234. gerade, die Schaulustige anzieht. Bei der Frage, um welche Hilfeleistung es konkret geht, die hier unterlassen wurde, müssen zwei Fälle unterschieden werden: Der des "schlechten Samariters" und der des typischen Schaulustigen..$^{59}$ Dies sei am Beispiel eines Verkehrsunfalls verdeutlicht. Der typische Anwendungsfall für den »schlechten Samariter « liegt darin, dass ein Verkehrsteilnehmer am Unfallort anhält, um einen Blick auf das Geschehen zu erhaschen oder Fotos schießen und dort - obwohl noch keine anderen Ersthelfer die Verletzten versorgen - darauf verzichtet, etwas zu deren Rettung zu unternehmen. Die erforderliche Hilfeleistung gem. § 323c StGB liegt hier darin, dem Verletzten aktiv Hilfe zuteil werden zulassen, beispielsweise durch das Verständigen von Rettungskräften oder die Durchführung von Erste-Hilfe-Maßnahme. Anders hingegen der klassische Schaulustige: Hier liegt häufig eine Situation vor, in der die Verletzten bereits ausreichend durch Erst-Helfer versorgt werden. Da somit bereits Hilfe geleistet wird, kommt es auf eine zusätzliche Hilfeleistung des Schaulustigen nicht mehr an..$^{60}$ Eine Hilfeleistung gem. § 323c StGB kann aber in jedem Tun und Unterlassen bestehen, so auch lediglich darin, den Weg für Rettungskräfte freizugeben..$^{61}$ Das Nichtbeiseitetreten kann also objektiv den Tatbestand der Unterlassenen Hilfeleistung erfüllen. ${ }^{62}$ Allerdings ist $\S 323 \mathrm{c}$ StGB ein Vorsatzdelikt. Der Täter müsste zunächst die Gefahrenlage, also die tatbestandsmäßige Situation als solche erkannt haben, was regelmäßig der Fall sein wird. Er müsste überdies aber auch erkannt haben, dass sein Nichtbeiseitetreten das Unterlassen einer erforderlichen Hilfeleistung darstellt. $\mathrm{Ob}$ eine Handlung wie hier das Beiseitetreten erforderlich ist, bestimmt sich nach dem ex-ante-Urteil eines verständigen Beobachters in der Person des Täters. ${ }^{63}$ Wenn damit zwar die große Gruppe der Schaulustigen in ihrer Gesamtheit die Anfahrt eines Rettungswagens behindert, so muss der einzelne Täter um § 323c StGB zu erfüllen, erstens erkannt haben, dass ein Beiseitetreten zur Abwendung des Unglücksfalls erforderlich ist und zweitens in diesem Wissen

59 Vgl. zu ersterem Schwind, Alle Gaffen - keiner hilft: Unterlassene Hilfeleistung bei Unfällen und Straftaten, 1998.

60 Anders nur, wenn dieser im Vergleich zu den vorhandenen Ersthelfern überlegenes Können und Wissen zur Abwendung der Gefahr mitbringt, wie z. B. ein Arzt, vgl. Kühl, in: Lackner/Kühl, StGB, § 323c Rn. 6.

61 Scheffler NJW 1995, 232, 234.

62 Schwind, Alle Gaffen - keiner hilft: Unterlassene Hilfeleistung bei Unfällen und Straftaten, 1998, S. 163, bezeichnet daher das Verhalten Schaulustiger im Vergleich zu dem Verhalten »Schlechter Samariter« auch als »abgeschwächte, quasi leichtere Form der Unterlassenen Hilfeleistung «.

63 Kühl, in: Lackner/Kühl, StGB, § 323c Rn. 5. 
auf seiner Position verharren. Die meisten Schaulustigen werden, sobald sie gewahr werden, dass sie einem Helfer im Wege sind, den Weg freigeben. In einzelnen Fällen werden besonders hartnäckige Schaulustige ihre Position nicht aufgeben oder später zurückkehren und damit erneut Rettungshandlungen behindern. Verurteilungen für das reine »Gaffen« sind auf Grundlage des § 323c StGB somit also grundsätzlich möglich, aber - soweit ersichtlich - bisher nicht ergangen. Das liegt vermutlich daran, dass erstens der Tatbestand der Unterlassenen Hilfeleistung in seiner Anwendbarkeit auf Schaulustige eher unbekannt ist und auch nur die oben beschrieben besonders renitenten Fälle erfasst. Zweitens haben die zuständigen Behörden in der Arbeit vor Ort häufig nicht die Zeit dafür, sich neben der Rettung Verletzter auch noch um die Strafverfolgung Schaulustiger zu kümmern. ${ }^{64}$ Dass »Gaffer» größtenteils unbehelligt bleiben, liegt damit vor allem an einem Vollzugsdefizit.

\section{VI. Überlegungen de lege ferenda}

Vor dem Hintergrund der besprochenen Probleme scheint es sinnvoll, den Schutz ziviler Helfer im StGB neu zu regeln. Zunächst ist eine Neuverortung der Regelung notwendig. Den Schutz professioneller Helfer als »Wurmfortsatz« des Widerstands gegen Vollstreckungsbeamte zu regeln, hat sich in mehrfacher Hinsicht als unglücklich erwiesen. Viele Probleme hängen damit zusammen, dass die Behinderung ziviler Helfer als $\S 114$ Abs. 3 StGB im komplizierten Verhältnis zu § 240 StGB seinen Platz gefunden hat. Als Vorschläge für eine Neuverortung werden in der Literatur § $145 \mathrm{StGB}^{65}$ und $\S 323 \mathrm{c} \mathrm{StGB}{ }^{66}$ genannt, die um einen entsprechenden Absatz ergänzt die Behinderung ziviler Helfer sachgerechter erfassen könnten. Eine Einordnung bei $\S 145 \mathrm{StGB}$, der den Missbrauch von Notrufen und die Beeinträchtigung von Unfallverhütungs- und Nothilfemitteln unter Strafe stellt, wäre hinsichtlich des geschützten Rechtsguts überzeugend. ${ }^{67}$ Eine Einordnung bei § 323c StGB wäre ebenfalls zu erwägen, da hier dieselben Indivi-

64 Vgl. Schlie, ZRP 2010, 129. 65 So Zopfs, GA 2012, 259ff., 264.

66 So wohl Wolters, in: SK-StGB, § 114 Rn. 9, der zu Recht auch die Frage stellt, warum der besondere Schutz für Hilfeleistende nur Helfern bestimmter Institutionen zu teil wird.

67 Als geschütztes Rechtsgut des $\S 145$ StGB gilt das »Allgemeininteresse an wirkungsvoller Hilfe in plötzlichen Notsituationen « und darüber hinaus "mittelbar [...] auch die von Notlagen bedrohten Rechtsgüter«, Fischer, StGB, § 145 Rn. 2. dualrechtsgüter geschützt werden, um die es auch bei § 114 Abs. 3 StGB mittelbar geht. Auch existiert bereits über die Merkmale »Unglücksfall, gemeine Gefahr oder Not« und den Begriff der Hilfeleistenden bzw. Hilfeleistung bereits eine inhaltliche Nähe zur Unterlassenen Hilfeleistung.

Darüberhinausgehend könnte der Schutz ziviler Helfer gemeinsam mit einem eingeschränkten »Gaffertatbestand « in einem neu zu schaffenden »§ 323d StGB - Behinderung von Rettungskräften« geregelt werden. Dieser könnte etwa wie folgt lauten:

(1) Wer bei Unglücksfällen oder gemeiner Gefahr oder Not Hilfeleistende der Feuerwehr, des Katastrophenschutzes oder eines Rettungsdienstes behindert, wird mit Freiheitsstrafe bis zu einem Jahr oder mit Geldstrafe bestraft.

(2) Erfolgt die Behinderung durch Gewalt oder durch die Drohung mit Gewalt oder durch einen tätlichen Angriff, so ist die Strafe Freiheitsstrafe bis zu drei Jahren oder Geldstrafe.

Die inhaltliche Nähe zu § 323c StGB würde so systematisch deutlich. In diesem Tatbestand könnten sowohl das »Gaffen « in Abs. 1 als bloßes Behindern der Rettungskräfte als auch das Behindern durch Gewalt oder durch die Drohung mit Gewalt oder einen tätlichen Angriff als Qualifikationstatbestand in Abs. 2 geregelt werden. Letzteres würde dann dem bisherigen § 114 Abs. 3 StGB entsprechen. Erreicht wären damit zwei Anliegen: Zum einen wäre die bisherige Lösung eines Tatbestands über den Schutz ziviler Einsatzkräfte nach $\S 114$ Abs. 3 StGB von seinen systematischen Problemen befreit. Zum anderen wäre mit $\S 323 \mathrm{~d}$ Abs. 1 StGB eine restriktive Lösung eines »Gaffertatbestandes" gefunden, dessen Anwendungsbereich nicht über den des $\S 323$ c StGB hinausreichen würde, der jedoch mit seiner klarstellenden Wirkung das Vollzugsdefizit der bisherigen rechtlichen Möglichkeiten ausgleichen könnte. $\S 323 \mathrm{~d}$ StGB würde neben den Individualrechtsgütern von Hilfsbedürftigen und Helfern auch die Rettungsbemühungen und -chancen schützen, die insbesondere durch den Abs. 1 in den Blick genommen werden. Nicht erfasst wären damit aber solche als »Gaffer-Situationen « diskutierte Fälle, in denen der Schaulustige nicht nur Rettungsbemühungen behindert, sondern selbst zur Gefahr wird, wie in dem oben beschriebenen Fall, dass schaulustige Autofahrer bei einem Verkehrsunfall auf der Gegenfahrbahn abbremsen und einen Stau verursachen. ${ }^{68}$ Hier liegt das Gewicht aber von vornherein auf der Herbeiführung einer neuen Gefahr und weniger auf der Behinderung der Beseitigung einer

68 Siehe oben $\mathrm{V}$. 
bestehenden Gefahr. ${ }^{69} \S 323 \mathrm{~d}$ StGB sollte allein an den Schutz von Helfern und Hilfeleistungen anknüpfen. Verursachen Schaulustige durch ihr Verhalten neue Gefahren, so müssen diese durch sachnähere Tatbestände, wie beispielsweise $\S \S 315$ b, c StGB erfasst werden.

\section{Fazit}

Das 44. StrÄndG hat mit der Erweiterung der §§ 305a, 114 StGB den Versuch unternommen, den Schutz ziviler Helfer zu verbessern. Der grundsätzlichen Intention des Gesetzgebers, Hilfeleistende strafrechtlich besonders zu schützen, kann dabei nur zugestimmt werden. Die Schutzbe-

69 Obgleich nicht ausgeschlossen ist, dass Rettungskräfte wie in dem beschriebenen Fall ihre Anfahrt zum Unfallort über die Gegenfahrbahn durchführen und daher durch eine neu entstandene Gefahr in ihren Rettungsbemühungen ebenfalls behindert werden. reichserweiterung in § 305a StGB ist dabei sinnvoll und angemessen. Nach Abzug eines möglicherweise symbolischen Teilgewinns ist aber zumindest im Hinblick auf $\S 114$ Abs. 3 StGB das Anliegen des Gesetzgebers fehlgeschlagen. Die Einordnung im Zusammenhang mit § 113 StGB stellt sich als systematisch verfehlt heraus, die Situation eines Angriffs durch den Hilfeleistungsadressaten selbst wird durch $\S 114$ Abs. 3 StGB nicht berücksichtigt und das substantivierte Merkmal "Hilfeleistende» bringt Auslegungsschwierigkeiten mit sich. Am schwersten wiegt jedoch, dass der Gesetzgeber durch die systematisch unbedachte Einordnung der Norm den Zweck seines Vorhabens gefährdet hat, da nach verbreiteter Ansicht für Angriffe auf zivile Helfer § 240 StGB nun gesperrt sein soll. Daher sollte eine Neuverortung vorgenommen werden. In einem $\mathrm{zu}$ schaffenden $\S 323 \mathrm{~d}$ StGB könnten sowohl Behinderungen der Rettungskräfte durch Schaulustige als auch in qualifizierter Form Angriffe auf deren Willensfreiheit und gegen deren Person sachgerecht erfasst werden. 